\title{
NOTA: a novel online teaching and assessment scheme using Blockchain for emergency cases
}

\author{
Anissa Cheriguene ${ }^{1,2} \cdot$ Taieb Kabache $^{2} \cdot$ Chaker Abdelaziz Kerrache $^{3}$ (D) \\ Carlos T. Calafate ${ }^{4}$ Juan Carlos Cano ${ }^{4}$
}

Received: 11 January 2021 / Accepted: 7 June 2021 / Published online: 3 July 2021

(C) The Author(s), under exclusive licence to Springer Science+Business Media, LLC, part of Springer Nature 2021

\begin{abstract}
Technology advancements promote a redefinition of traditional instructional methodologies, as well as the roles of teachers and learners towards an efficient e-learning ecosystem. To date, all existing solutions are combined with the conventional face-toface learning process. However, the latter can be unexpectedly hindered in some emergency cases, like the Coronavirus (COVID-19) pandemic. To handle such unexpected scenarios, this paper presents NOTA, a novel online teaching and assessment scheme that takes advantage of Blockchain technology to maintain the expected teaching quality and assessment fairness while respecting the courses' and examinations' schedule. Besides, NOTA also motivates both learners and teachers to persist in their endeavours, even from home, through Blockchain's incentive strategies. The preliminary results taken during the CoronaVirus period showed a very high satisfaction ratio, exceeding the $90 \%$. This made us feel very optimistic about the potential of our proposal when deployed at a larger scale.
\end{abstract}

Keywords Blockchain technology Educational technology $\cdot$ Education during pandemics - Distance learning · COVID-19 and education

\section{Introduction}

Computers and the Internet have become an indispensable part of human's personal and professional lives, and are quasi omnipresent in all domains. Throughout the years, the use of Information and Communication Technologies (ICT) has fundamentally altered the practices and procedures of nearly all forms of endeavors in business and governance. As far as education is concerned, methods of teaching and learning have

RESEARCH

NOTA: a Novel Online Teaching and Assessment scheme using Blockchain for Emergency Cases

Chaker Abdelaziz Kerrache

ch.kerrache@lagh-univ.dz

Extended author information available on the last page of the article 
considerably changed due to the adoption of technology. As such, ICT played an important role in modern education by promoting more independent, motivated, and autonomous learners. To this end, several online teaching (e-learning) solutions emerged (Kats, 2010). They are used as an important complementary tool that should work together with the conventional face-to-face classes.

It is asserted that the usage of ICT learning settings and instruments in educational processes shall denote radical changes both in the role of teachers and learners, leading to the upbringing of new teaching and learning environments and methodologies (eLearning, Web-based Learning, Open and Distance Learning), as well as new training modalities (on-line training, on-site training, Blended-Learning, Instructor led Learning/Training, and Classroom Training) (Pieri \& Diamantini, 2009).

Usually, e-learning methods are classified into Massive Online Open Course (MOOC), Web 2.0-based, and Blended Learning (BL) (Tirziu \& Vrabie, 2015). Yet, all three categories suffer from a number of drawbacks including: the lack of incentive strategies targeting both teachers and students, the lack of traceability, and many times an unfair assessment. This is why they are considered as complementary to the face-toface classroom teaching/training approach, but cannot replace it.

Furthermore, all previously mentioned solutions do not offer a universal platform that can be used by teachers, students, and institutions to ensure the continuity of the courses and assessments when facing an emergency case like the current pandemic caused by the Coronavirus (COVID-19) (Sahin et al., 2020). To face this virus, which affected and continues affecting many hundreds of thousands of people worldwide, one of the most effective solutions to date is to stay at home. Thus, the need for a new cooperative online teaching and assessment scheme for emergency cases became a priority.

Recently, Blockchain has emerged as a fully decentralized and auditable network where every participating node can add reliable/verifiable data to the blockchain, thereby providing a security-by-design architecture (Swan, 2015). Blockchain was originally designed for the financial industry, which uses cryptocurrencies such as Bitcoin (Crosby et al., 2016), and it is currently revolutionizing the IoT industry as well, including healthcare (Mettler, 2016), supply chain (Abeyratne \& Monfared, 2016), and logistics (Dobrovnik et al., 2018). However, existing works focus mainly on the use of blockchain to ensure the e-learning systems access control and certification, but not for the teaching and assessment process (Tolbatov et al., 2018; Ma et al., 2018; Lam \& Dongol, 2020; Awaji et al., 2020).

In this paper we propose a novel online teaching and assessment scheme called" NOTA". Our proposal takes advantage of the Blockchain technology to offer a fully distributed and secure educational system for emergency cases. NOTA proposes the use of three universal Blockchains for course deployment, course completion, students examination, and finally for the assessment of the students responses.

The rest of the paper is organized as follows: In Section 2, we provide an overview of the existing Online teaching and assessment solutions, as well as the main principles of Blockchain technology. Section 3 introduces our proposed architecture (NOTA), and its details are discussed in Sect. 4. Section 5 evaluates NOTA's performance, and the advantages of using Blockchain are discussed in Sect. 6. Finally, Sect. 7 concludes the paper. 


\section{Background and related work}

This section discusses some of the existing e-learning solutions. In addition, it also provides an overview of Blockchain technology, on which our proposal is based.

\subsection{Existing e-learning approaches}

As mentioned above, e-learning solutions are usually classified into MOOC-based, Web2.0-based, and blended learning solutions. In the following we discuss the main existing works in each category.

\subsubsection{MOOC-based solutions}

Considered as one of the most recent trends in education, the term MOOC represents open access, free video-based content through an online platform addressing a high volume of participants who aim at taking a course, or simply getting an education (Haumin \& Madhusudhan, 2019).

MOOCs have an obvious capacity to scale, providing access to a vast number of users worldwide to attend free online classes, beyond the borders and formalities of traditional education (Yuan \& Powell, 2013). They have unique features that are able to make a movement toward the relation of lifelong and on-demand learning for those who are working full time, or have taken a break from formal education (Yousef et al., 2015). By offering time and place flexibility, MOOCs such as Coursera ${ }^{1}$, Udacity ${ }^{2}$, Udemy $^{3}$, and Edraak ${ }^{4}$ are considered to be an endowment for the present generation, enabling education to achieve a degree of worldwide penetration that was never thought to be possible decades ago. However, these solutions can be mostly considered as a training rather than courses, and they cannot replace the conventional educational system during emergency situations.

\subsubsection{Web2.0-based solutions}

Indeed, Web 2.0 platforms or instruments like online discussion, forums, blogs, Flicker, Instagram, Twitter and many more are thought to have came out with a critical role to change the path of teaching and learning processes. These instruments allow users to communicate and collaborate with each other via social media in a virtual community. The incorporation of web 2.0 resources in education became a trend as it transformed the existent educational landscape, enabling information sharing and facilitating discussion among online learners.

Researchers have been and are still interested in the use of web 2.0 technology in educational settings. Greenhow et al. (2009) demonstrated how the unique abilities of the web 2.0, combined with the youth's proclivities in using it, can affect the teaching and learning processes. In particular, two main topics emerged from their study: (i)

\footnotetext{
${ }^{1}$ https://fr.coursera.org/

${ }^{2}$ https://www.udacity.com/

${ }^{3}$ https://www.udemy.com/

${ }^{4}$ https://www.edraak.org/
} 
learner participation, and (ii) creativity and identity formation. Yunus et al. (2012) discussed how the use of Facebook groups can enhance students' writing. They concluded in their study that Facebook can be an effective tool in teaching ESL writing, especially at involving the tertiary level. In the same line, Manca \& Ranieri, 2016 wrote a critical review of different studies, elaborating on how Facebook is being used as an instrument in a technology- based learning environment, with the purpose of figuring out how far do we actually turn its pedagogical potential into practice. Similarly, the authors of (Doolittle \& Hicks, 2003) summarized in an article the theoretical foundations of using social networking in language classrooms, and in what ways the tools offered in social networking can be effective in the learning process. Moreover, the paper by Shariffuddin et al. (2017) intended to study the benefits and drawbacks of emerging social networking instruments in ESL classrooms, particularly in writing lessons, and propose ways to design activities by including Social Networking Services (SNSs) in the teaching and learning sphere.

\subsubsection{Blended learning solutions}

Blended learning, also known as "hybrid learning", is defined as "the concept that includes framing teaching learning process that incorporates both face-to-face teaching and teaching supported by ICT" (Stevenson, 2018). It is a combination of some traditional classroom characteristics along with an online insertion of specific content to specific learners, including face-to-face classroom lessons, live e-learning, and selfpaced learning.

Blended learning was first exploited in the corporate world as a method to provide access to workers to work and study at the same time (Thorne, 2003). According to Thorne, 2003, blended learning has attracted different definitions and stances. The concept blend has been used to refer to not only the mix of two teaching modes, but also to the combination of technologies (email, phone, web, etc.) or methodologies (presentation, practice, production). Researchers define blended learning as a mixture of online learning or web-based training with face-to-face contact, and traditional ways of learning and teaching (Sharma, 2010; Chandra, 2004; Graham, 2006; Staker \& Horn, 2012).

The most important aim of blended learning, however, is to create an inviting learning environment that incorporates the best of both modes, and that functions as a whole to find "the most effective and efficient combinations of the two modes of learning for the individual learning subject contexts and objectives" (Neumeier, 2005). Blended learning seeks to create a harmonious and cohesive balance between online access to knowledge and face-to-face interaction by taking into account learners' and teachers' aptitudes and attitudes. Hence, blended learning establishes itself as a significant term in education as "its overall focus is concerned with the attempt to identify the optimum mix of course delivery in order to provide the most effective language learning experience" (Sharma, 2010).

\subsection{Blockchain technology: An overview}

Blockchain is a distributed ledger system which allows multiple users to securely store, process and share data in decentralized and peer-to-peer network settings (Swan, 2015). It gets its name from the fact that transactions are stored in blocks which are linked with 
each other to form a chain. A block contains various information about a transaction, including the time of the transaction, the sequences of transactions, and a pointer to the previous block. Indeed, linking transaction blocks using a hash will ensure the integrity of data on each transaction (previous blocks cannot be altered), and prevent the insertion of new blocks in-between two pre-existing blocks.

In addition, to ensure full data integrity and availability in the blockchain, each blockchain platform must guarantee the key blockchain concepts:

- Data immutability: The notion that once data is written and stored, it cannot be deleted or altered. This key concept is one of blockchain's main benefits since it develops trust between blockchain members.

- Shared ledger: a network where all members have an immutable copy of all transactions. In a shared ledger, transactions are only recorded once to avoid any duplication, but this ledger is permissioned so that members can only see what they are authorised to.

- Consensus: a mechanism used to achieve agreement on a piece of data be- tween all members of the network. Different algorithms have been developed to ensure this key feature, such as Proof-of-work, where specific nodes, referred to as miners, compete against each other to complete transactions on a network for a reward. The transaction is then independently verified by all nodes on the blockchain before it is added to the blockchain.

- Permissions: blockchains can either be permissioned or non-permissioned, meaning that members of a blockchain either have restricted access on a blockchain or do not.

- Cryptography: cryptography is used to hash transactions and store them in blocks so that data is protected, and can only be read by authorised users. This is a method of encrypting and decrypting information through complex computation. It is a key aspect of blockchain, as it is used to protect user's personal data, as well as ensuring that data stored in blocks are secure (Ahmad et al., 2019).

The main advantage of a public blockchain is its autonomy. All users have similar privileges, and no party can control stored data, which means that users do not have to trust and rely on a third party. However, public blockchains are extremely large and consume a large amount of energy as no user has access restrictions. On the other hand, private Blockchains tend to be smaller and flexible, as only a limited number of users are allowed access, and they have different permissions and access privileges.

\section{NOTA: A bird's eye view}

In this section we provide an overview of the architecture of NOTA, as well as the main functionalities offered by our novel solution.

\subsection{Architecture of NOTA}

NOTA is composed of three main domains: (i) Teaching domain; (ii) Interactions middleware; and (iii) Blockchain-enabled Infrastructure domain. Next, we explain the tasks of these three domains, as illustrated in Fig. 1. 


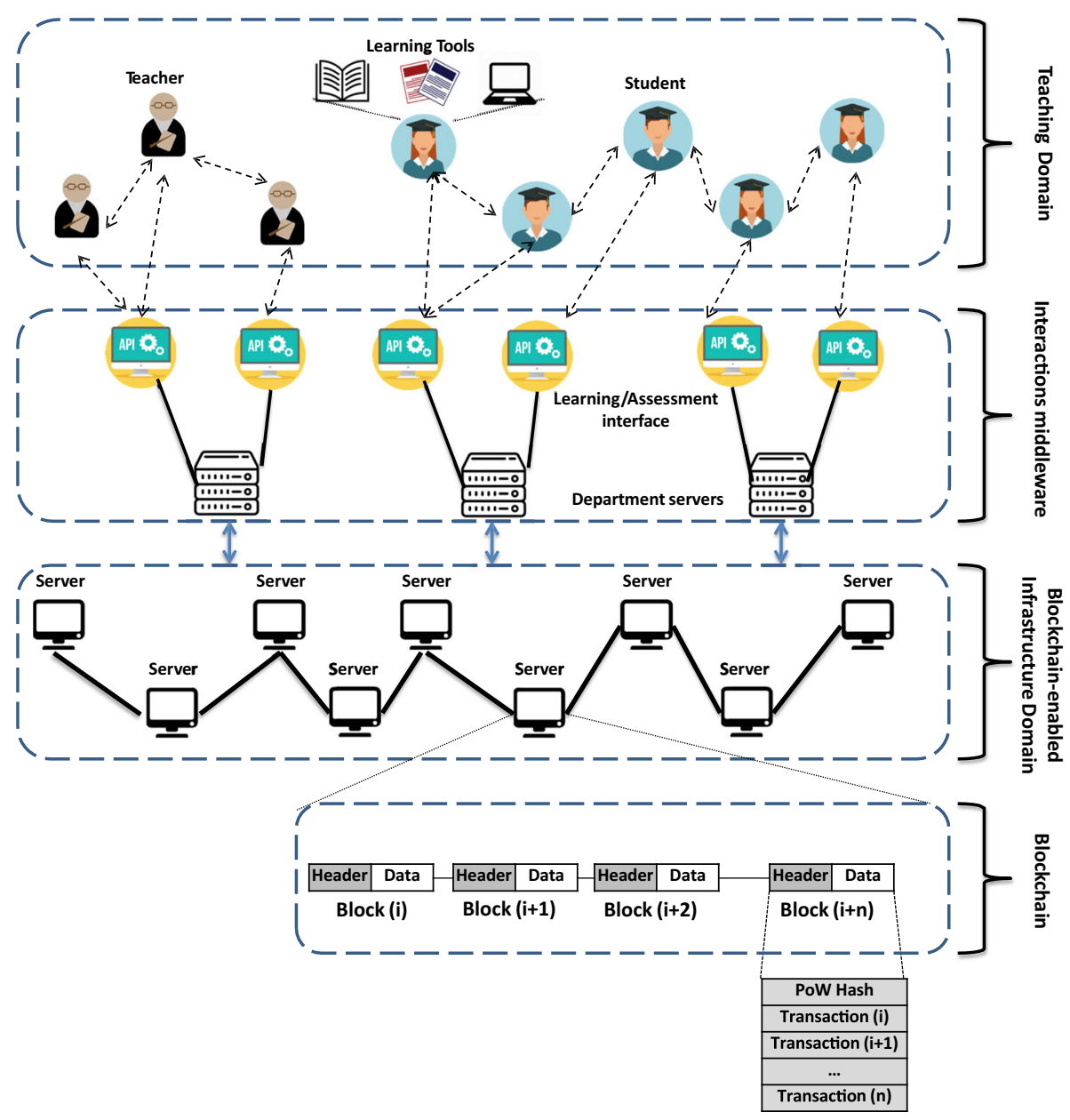

Fig. 1 Proposed NOTA architecture using Blockchain

\subsubsection{Teaching domain}

This domain is composed of the main two actors of the educational system, learners and teachers. It also includes the the additional means of learning that can be used by learners outside the online course including books, handouts, and Internet documentation. Besides, same as the conventional case, here learners can exchange and discuss their courses through the different communication technologies and social networks.

\subsubsection{Interactions middleware}

This domain representing the communication middleware is responsible for sharing the message originated from both learners and teachers with the backend infras- tructure through a dedicated interface accessible via previously given credentials than ensure role differentiation (i.e., a different interface for 
learners and teachers). Furthermore, department servers in this domain can act as cluster heads to transmit information towards the back-end network in order to record data into the Blockchain.

\subsubsection{Blockchain-enabled infrastructure domain}

This domain is the back-end domain of the NOTA architecture, which is equipped with Blockchain. Miners are first elected in this domain based on their capability to solve the complex Proof-of-Work (PoW) puzzle, which will also be the subject of an incentive (reward) later on. This PoW represents the consensus algorithm of the Blockchain explained above (Baliga, 2017).

For the NOTA architecture we distinguish between three blockchains, namely OCER, SR, and AR: (i) Online Courses and Exam Registration (only teachers are allowed to add transactions to this blockchain), (ii) Students' Responses registration (only students are allowed to add transactions to this blockchain), and (iii) Assessment Registration (only teachers are allowed to add transactions to this blockchain). When the data from the student/teacher is received at the miners via the communication domain, the Miners first validate the information based on the consensus algorithm. Once the PoW puzzle is solved by the miner, the data can be added within the Blockchain. The block in NOTA has two major components, i.e., (1) Block header, and (2) Block data. Block header contains the Proof-of-Work Hash, which includes control-related information such as version number, Nonce value, previous block information, merkle trees, and a timestamp. Block data contains the list of the transactions which are recorded by the miners after solving the PoW algorithm.

\subsection{NOTA functionalities}

NOTA takes advantage of blockchain to provide a secure and timely connectivity between students, teachers, and their departments. It operates in two main phases:

- First, system actors (students and teachers), through dedicated interfaces (APIs), login and register the different allowed actions. This information can contain sensitive data, including students/teachers identities, scores, and contacts.

- Second, the miners at the Blockchain-enabled Infrastructure domain can add the information into the Blockchain after solving the PoW algorithm. Miners are the only nodes responsible for adding the data into the Blockchain. However, the data is not added to the Blockchain if a miner is unable to solve the PoW consensus algorithm. Unlike the conventional case (applied for the courses and student responses' Blockchains), we allow three miners to add data into the same block in the assessment Blockchain, which represents three different evaluations of a given student response. This a configurable parameter that can be used in different scenarios where, for example, we want to obtain a fair evaluation in particular exams with few enrolled students. In this case we need to obtain a fair classification of students' performance. 


\section{NOTA details}

In emergency scenarios where learners and teachers are compelled to stay at home, blockchain technology can be employed to provide a revolutionary distance-learning strategy. It takes advantage of the Blockchain's secure open ledger to give remote access to courses and tests to both students and teachers throughout the world.

NOTA has two main actors "Teachers" and "Students". In addition, it also involves dedicated interfaces for both actors, department servers, and the three Blockchains: (i) "Blockchain CEx" for courses and exams deployment by teachers, (ii) "Blockchain S" for course attendance and responses to the exams by the students, and finally (iii) "Blockchain Ev" for the registration of the evaluation of the students' responses by the teachers. The following subsections provide the details of each of the above tasks.

\subsection{Courses and exams registration by the teachers}

The first task to be completed in NOTA is the courses and exams deployments by the teachers. Same as all online systems, teachers should pass by an authentication phase before the courses and exams registration in the Blockchain CEx. Before finalizing this task, the department servers should run the Proof-o-Work/Proof-of- stake algorithm and solve the complex puzzle. Once done, a new block containing the new course with its exam will be included in the Blockchain CEx, and then a reward should be appended to the teachers account. Many forms of rewards (incentive strategy) can be used here including:

- Extra points for the promotion.

- Payment vouchers.

- Payment of conferences registration fees.

- Travel grants.

- Etc.

At a certain stage, the department can also disincentive a teacher refusing to deploy the courses and exams by a given deadline. Figure 2 summarizes the courses and exams deployment process to be done by the teachers.

\subsection{Courses attendance and responses to the exams by the students}

The most important actor of any education system is indeed the student. Same as their teachers, students should pass by an authentication phase prior to accessing the course materials. Afterwards, they select the target course, which will be brought to them from the "Blockchain CEx". After completing the course they can also access the exam, which will trigger a timer for the specified exam duration.

Once the responses are sent back by the student, or the time response elapses, the responses will be added to the "Blockchain $\mathrm{S}$ " after a mining process done by the department server. A notification of reception confirmation is then sent back to the student, as illustrated in Fig. 3: 


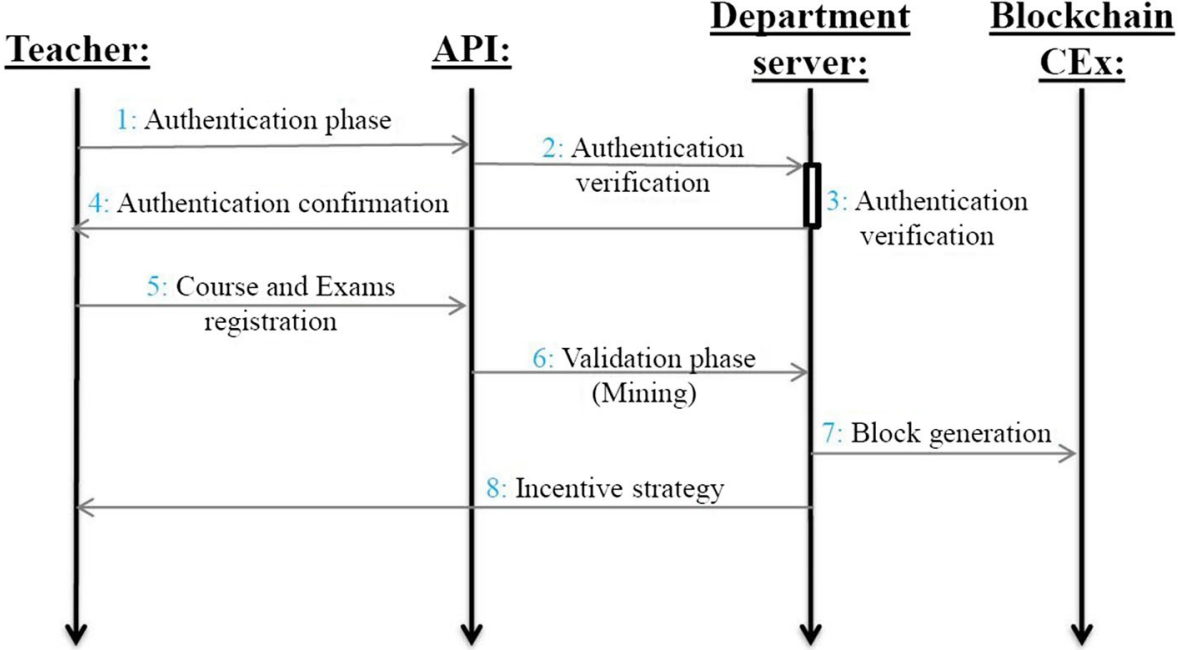

Fig. 2 Courses and exams registration in NOTA

\subsection{Evaluation of the students' responses by the teachers}

After receiving students' responses, the final task is the evaluation registration. Unlike the conventional case, a block of the "Blockchain Ev" here has three different records attached to the same responses' sheet of a given student. This is to ensure a fair evaluation of students responses, which will receive up to three different evaluations by three different teachers when needed.

Besides the case of option-based exercises where the evaluation is automated, and after passing the authentication phase, a teacher selects the course from the "Blockchain CEx" through the department's server. The latter will automatically provide the teacher with the responses sheet of an anonymous student that has completed the selected

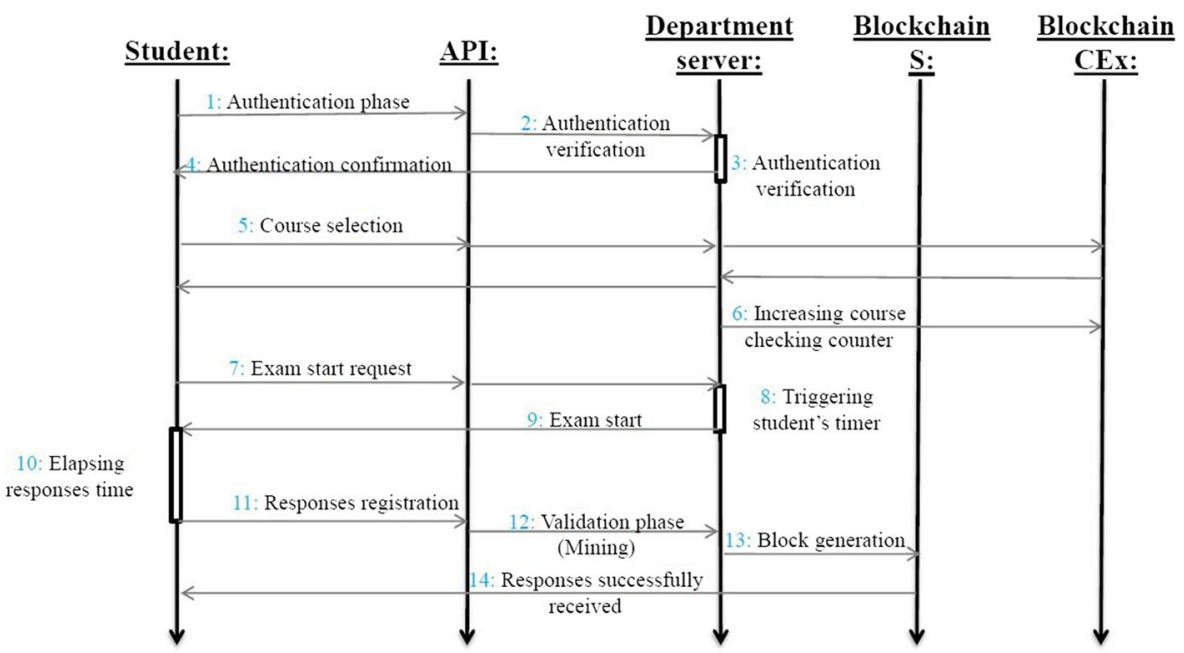

Fig. 3 Lectures and exams in NOTA 
course. Once the evaluation is done by the teacher, the score is recorded in "Blockchain Ev". Afterward, the system provides the teacher with another anonymous response sheet. As we mentioned above, the system makes sure that every student's sheet receives three different scores (from three different teachers) (see Fig. 4).

\section{Performance evaluation}

Since all the information is stored on the Blockchain, which provides privacy, reliability, and data immutability, a Blockchain-enabled education system can address the target requirements. Indeed, by implementing a permissioned Blockchain network like MultiChain or Fabric, only the university authorities will be able to add and maintain student information. Everyone, however, would have read access to the records.

To evaluate the performance of NOTA, we implemented the blockchain-related actions (i.e., blockchain creation, validation and insertion processes) in Java over using the Ethereum Proof-of-Work platform. Figures 5 and 6 illustrate the used code for both data insertion and mining processes.

\subsection{Blockchain-related performance}

To assess the impact of using Blockchain on the resources of the department's servers, we measured the average consumed energy per hour for the three main servers in the Department of Mathematics and Computer Science in the University of Ghardaia, Algeria. Besides the conventional tasks, these servers play the role of miners when using blockchain technology.

Figure 7 shows a comparison between the average consumed energy by these servers with and without Blockchain. It shows that, by using Blockchain, the servers consumed $15 \%$ more energy than the conventional situation. Yet, this additional overhead is still reduced compared to the advantage of such fully distributed educational scheme. Besides, when implementing the full scheme at a regional or a national scale, the cost will be significantly reduced as all computations will be running on the edge of the network.

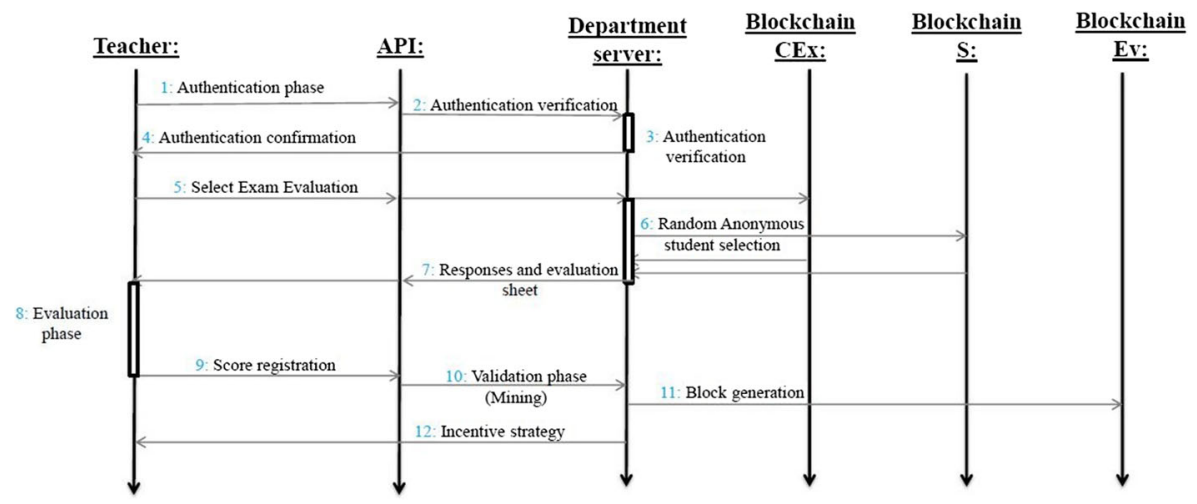

Fig. 4 Assessment procedure in NOTA 


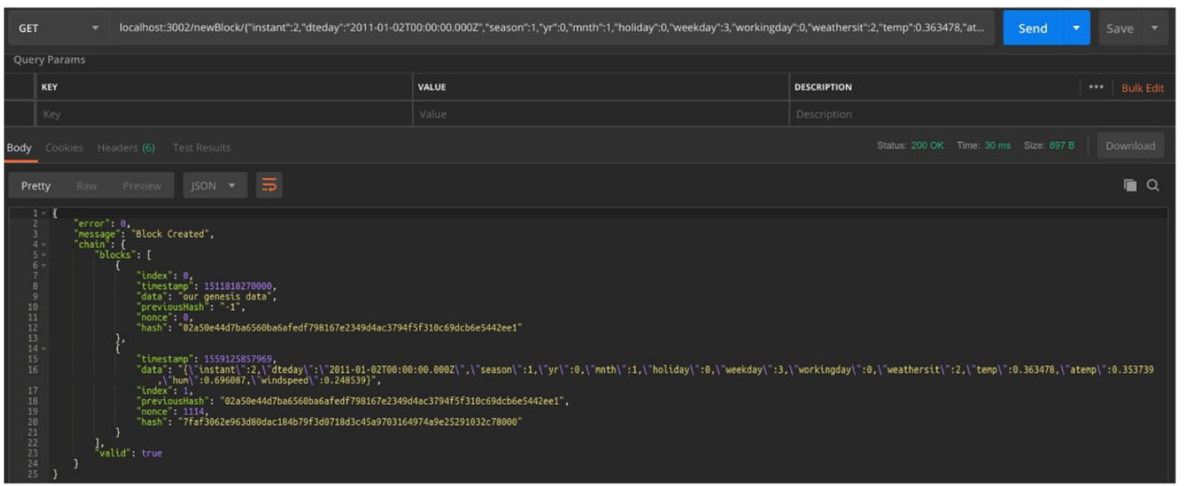

Fig. 5 Data insertion procedure

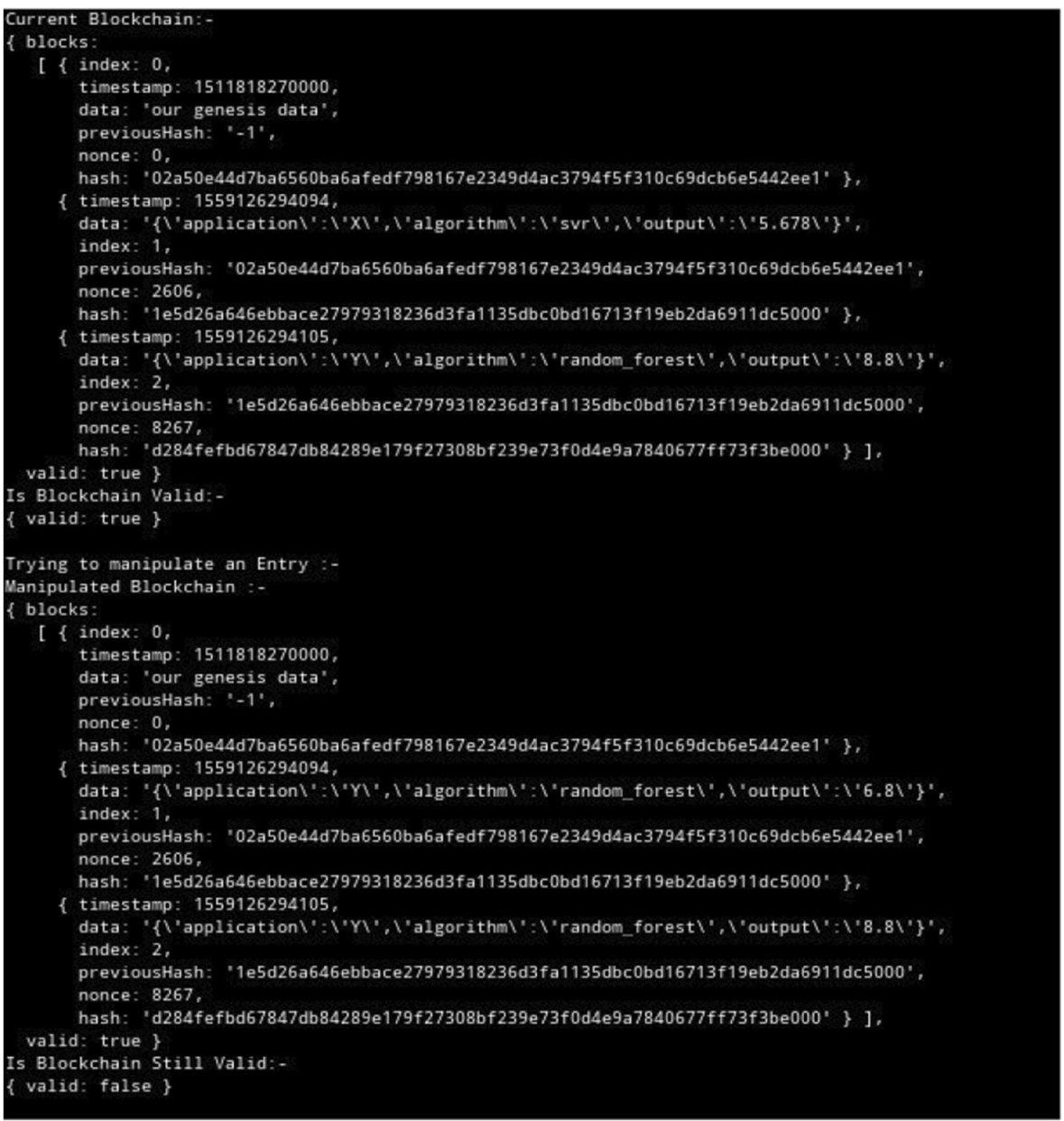

Fig. 6 Mining process 


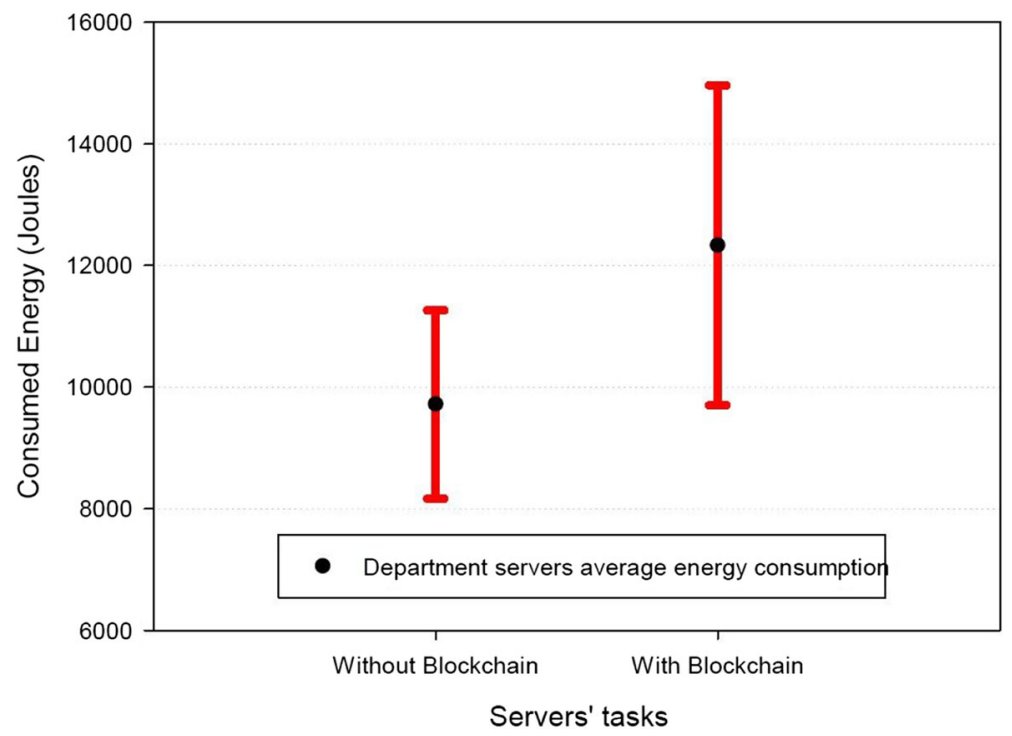

Fig. 7 Department servers energy consumption with and without Blockchain

\subsection{Online course performance}

Besides, we tested the effectiveness of our online learning/assessment by involving 211 students from which 21 are second year master students, 40 are first year master students, 62 are third year bachelor students, and 88 are second year bachelor students, all of them from the department of Mathematics and Computer Science at the University of Ghardaia, Algeria. We also involved five teachers, two of them from the École Normale Suṕerieure (ENS) of Laghouat, and three from the University of Ghardaia, Algeria. Students have online access to the courses of Scientific Research Methodology (2nd year master students), Network Administration (1st year master students), Cryptography (3rd year bachelor students), and Communication Networks (2nd year bachelor students); once they feel ready, they can start an online exam of three exercises (every exercise is defined by a different teacher) for the duration of $1 \mathrm{~h}$. Afterwards, the three teachers evaluate the anonymous responses of the students, and register the scores in the system.

\subsection{Assessment error ratio}

After collecting three different assessments for every student's response sheet, we measured the variation of these evaluations.

For the sake of simplicity, we visualize the results of the first 12 students. Figure 8 shows the confidence interval for every student's score based of three different anonymous evaluations. It depicts that, besides the student ' 9 ' which received a plus $(+)$ or minus (-) two points variation, all other evaluation variations were very reduced, not exceeding one point in the worst case. Students ' 6 ' and ' $10^{\prime}$ ' received exactly the same score by all three instructors. If we assume that a two-points variation is a fail, 


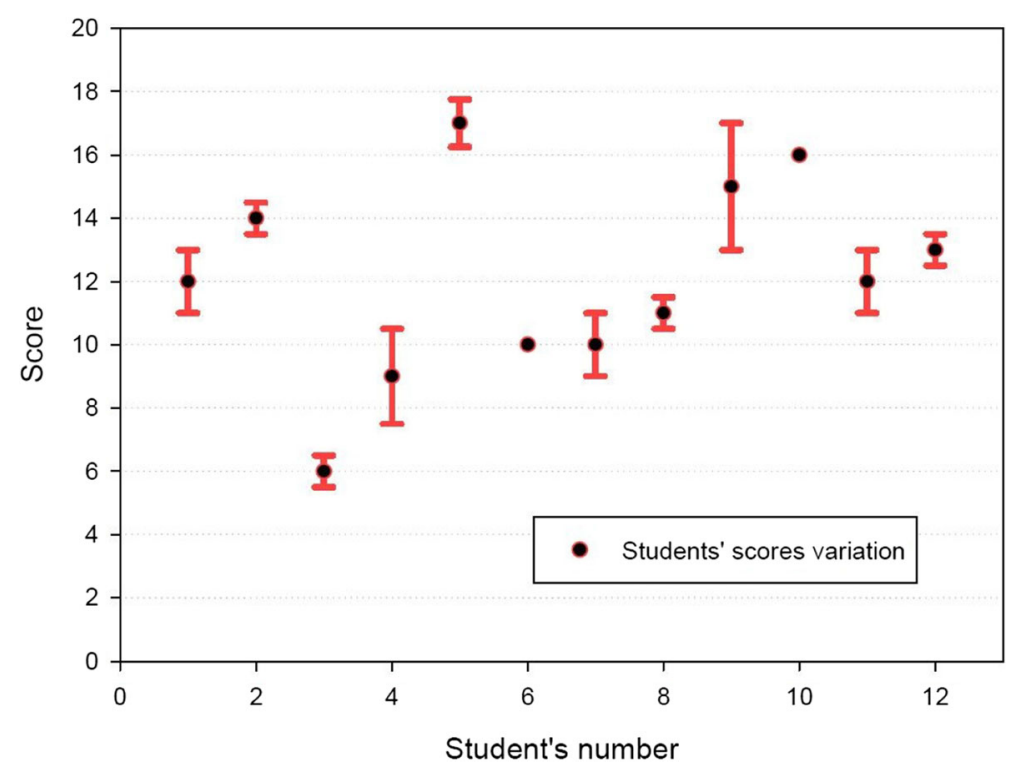

Fig. 8 Evaluation variation

then the success of our NOTA scheme in terms of evaluation exceeds the $91 \%$ for only 12 students. This ratio will be clearly increased for a higher number of students.

\subsection{Students satisfaction ratio}

Since students are the main actor of any educational system, we also collected the opinions of all students participating in the online course, and classified these opinions into three categories: Satisfied, unsatisfied, or partially satisfied. The received feedback is presented in Fig. 9. It is clear from the illustrated results that 167 students $(79.15 \%)$ were satisfied with this method involving different course materials, and different evaluations from various teachers, whereas 44 students $(20.85 \%)$ were unsatisfied or partially satisfied. When matching these latter opinions with their obtained scores, we found that they belong to the students receiving low scores, which partially explains their dissatisfaction.

\subsection{Teachers satisfaction ratio}

We repeated the same satisfaction queries with the teachers this time. The received feedback is illustrated in Fig. 10, where four teachers were satisfied with the overall NOTA system, whereas one teacher was partially satisfied. However, the latter also mentioned that he is not comfortable with all ICTbased teaching, not only NOTA. Finally, to measure the incentive variation, and to see who are the most contributing teachers, we gave one point for every completed task by a teacher. Figure 11 shows the the variation of incentive among teachers for the different courses which will help when providing the different kinds of incentives. 


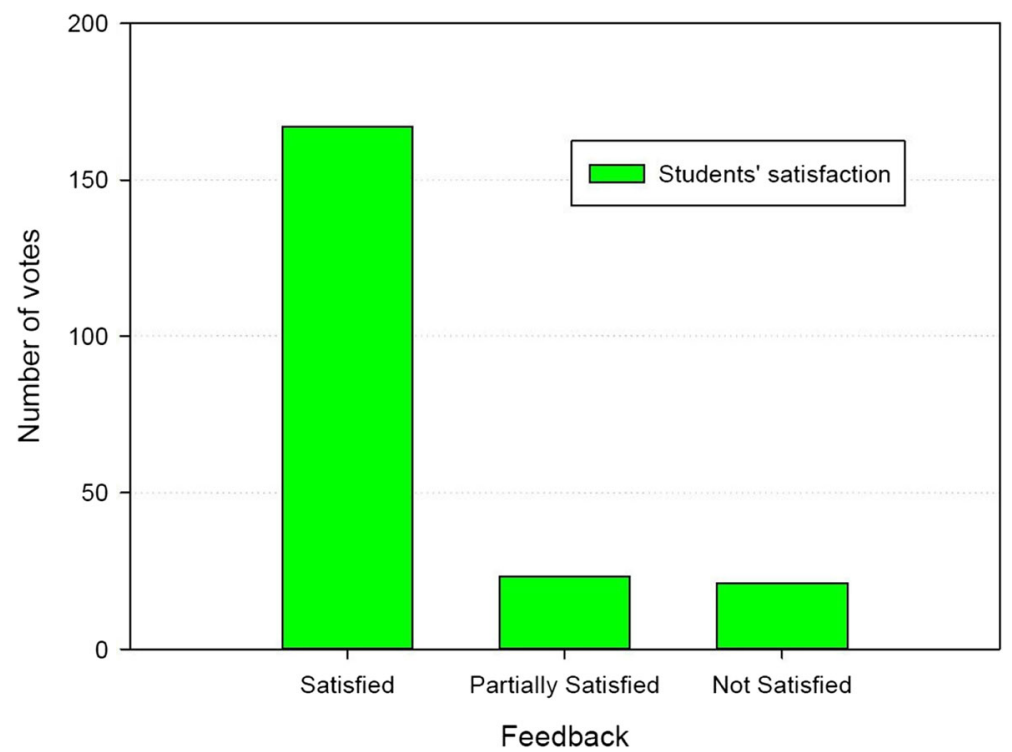

Fig. 9 Students' satisfaction of NOTA

\section{Advantages of using Blockchain technology for educational purposes}

Several new applications emerged along with Blockchain, including educational applications, that have many advantages such as:

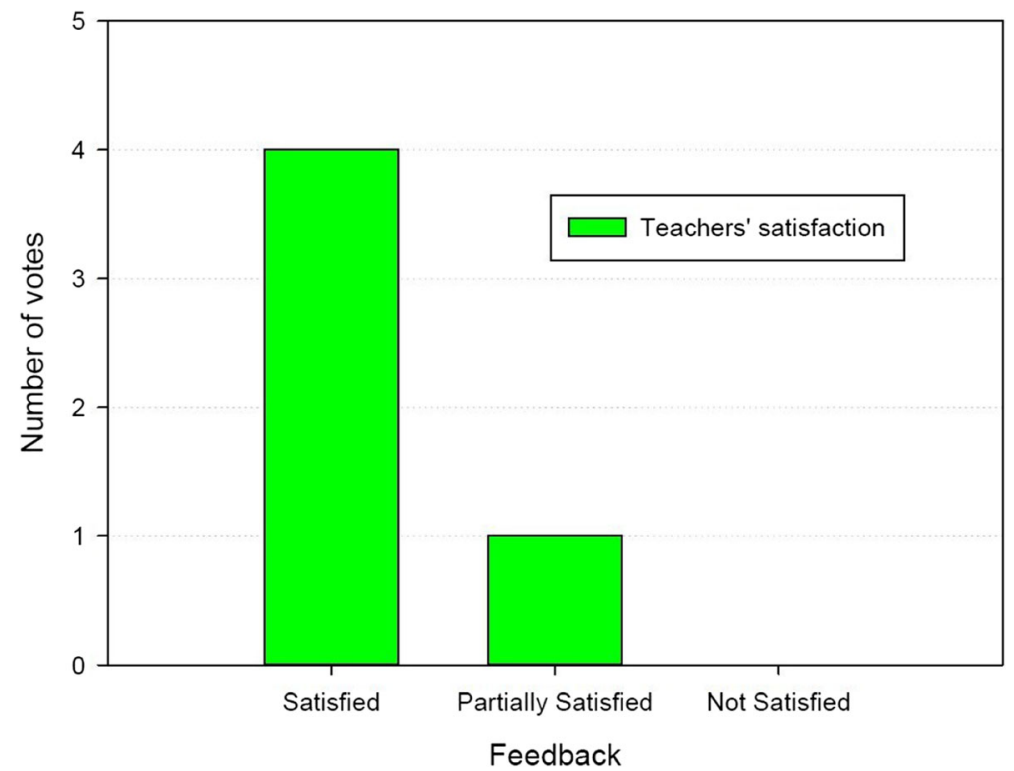

Fig. 10 Teachers' satisfaction of NOTA 


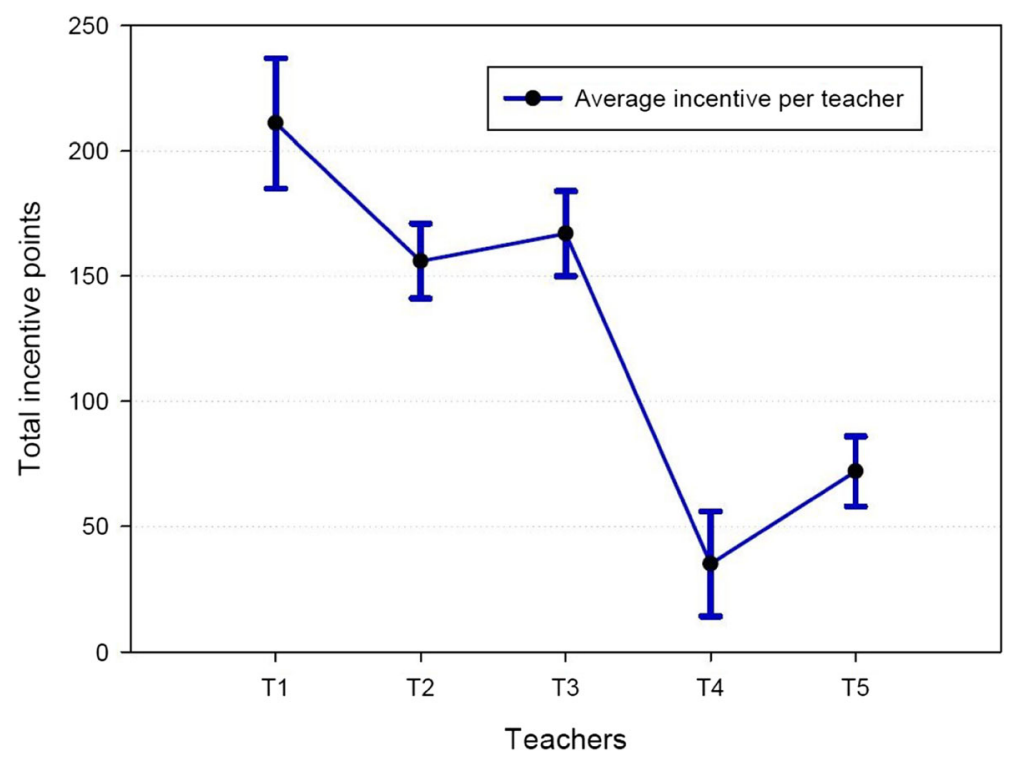

Fig. 11 Teachers' incentive in NOTA

- Worldwide courses can be accessed anytime and anytime; thus, both students and teachers will always be updated.

- Responses to the exams and scores will always be available, and cannot be changed.

- The incentive mechanism will encourage teachers to work more, and to be more productive.

- Students applications' to a new degree will be more fluent as all their information and curriculum are already available on the blockchain.

- In disaster cases, like the pandemic situation due to corona-virus (COVID- 19), students, teachers, and administration stuff can still work in a completely trusted, fair, and secure platform while in quarantine, as per government requirements.

\section{Conclusions}

The availability of the Internet has reinforced the combination of many existent multimedia services as tools for creating a Virtual Learning Environment (VLE) that asserts an enhanced content management, a better teaching environment, and reestablished the active role of the learner. This paper takes advantage of Blockchain technology to present a novel distance-learning solution for emergency cases where learners and teachers are forced to stay at home. It uses the features of Blockchain's secure open ledger to provide a distant access to the courses and exams to both students and teachers worldwide. Our proposal also incentives the efforts of the teachers preparing courses materials and participating in the evaluation process. Hence, it motivates them to keep working while in quarantine. The experimental results obtained show a high satisfaction ratio from both teachers and students.

As future work, we plan to add further functionalities to NOTA including distance supervision and tutoring, dissertations defense, and lab sessions. We also plan to develop 
an intuitive API interface that facilitates the different NOTA tasks. After- wards, making NOTA a smartphone Android/IOS application is also among our priorities.

Abbreviations APIs, Application programming interfaces; AR, Assessment registration; COVID-19, Coronavirus disease 2019; ESL, English as a second language; ENS, Ecole Normale Suṕerieur; ICT, Information and communication technologies; MOOC, Massive online open course; OCER, Online courses and exam registration; PoW, Proof-of-work; SNSs, Social networking services; SR, Students' responses registration

Acknowledgements Authors would like to acknowledge and thank the five teachers as well as the 211 students for all the information over which the evaluation of this research work has been conducted. We also would like to thank the University of Ghardaia in Algeria, and the Universitat Politecnica de Valencia in Spain, for supporting the undertaking of this work.

Author contribution Authors equally contributed to this manuscript.

Funding This research did not receive any kind of funding.

Data availability Full examination sheets and students scores for the different courses will be publicly provided through "https://www.univ-ghardaia.dz/" upon finalizing of all pedagogical activities. However, students and teachers names will not be revealed because of the university's privacy policy.

\section{Declarations}

Conflict of interest Authors declare no competing interests.

\section{References}

Abeyratne, S. A., \& Monfared, R. P. (2016). Blockchain ready manufacturing supply chain using distributed ledger. International Journal of Research in Engineering and Technology, 5(9), 1-10.

Farhan Ahmad et al. (2019). Blockchain in internet-of-things: Architecture, applications and research directions. In: 2019 International conference on computer and information sciences (ICCIS), pp. 1-6. IEEE.

Bakri Awaji, Ellis Solaiman, and Adel Albshri (2020). Blockchain-based applications in higher education: A systematic mapping study. In: Proceedings of the 5th international conference on information and education innovations, pp. 96-104.

Baliga, A. (2017). Understanding blockchain consensus models. Persistent, 4, 1-14.

Vinesh Chandra (2004). The impact of a blended web-based learning environment on the perceptions, attitudes, and performance of boys and girls in junior science and senior physics. PhD thesis. Curtin University.

Crosby, M., et al. (2016). Blockchain technology: Beyond bitcoin. Applied Innovation, 2.6-10, 71.

Dobrovnik, M., et al. (2018). Blockchain for and in logistics: What to adopt and where to start. Logistics, 2(3), 18.

Doolittle, P. E., \& Hicks, D. (2003). Constructivism as a theoretical foundation for the use of technology in social studies. Theory \& Research in Social Education, 31(1), 72-104.

Charles R Graham (2006). Blended learning systems. In: The handbook of blended learning: Global perspectives, local designs, pp. 3-21.

Greenhow, C., Robelia, B., \& Hughes, J. E. (2009). Learning, teaching, and scholarship in a digital age: Web 2.0 and classroom re- search: What path should we take now? Educational Researcher, 38(4), 246-259.

Lun Haumin and Margam Madhusudhan (2019). An Indian based MOOC: An overview.

Yefim Kats (2010). Learning management system technologies and software solutions for online teaching: Tools and applications: Tools and applications. IGI Global.

Tsz Yiu Lam and Brijesh Dongol (2020). A blockchain-enabled e-learning platform. In: Interactive learning environments, pp. 1-23. 
Sihua Ma et al. (2018). Using blockchain to build decentralized access control in a peer-to-peer e-learning platform. PhD thesis. University of Saskatchewan.

Manca, S., \& Ranieri, M. (2016). Is Facebook still a suitable technology-enhanced learning environment? An updated critical review of the literature from 2012 to 2015. Journal of Computer Assisted Learning, 32(6), 503-528.

Matthias Mettler (2016). Blockchain technology in healthcare: The revolu- tion starts here. In: 2016 IEEE 18th international conference on e- health networking, applications and services (Healthcom), pp. 1-3. IEEE.

Neumeier, P. (2005). A closer look at blended learning-parameters for designing a blended learning environment for language teaching and learning. ReCALL, 17(2), 163-178.

Michelle Pieri and Davide Diamantini (2009). From e-learning to mobile learning: New opportunities. In: Mobile learning: Transforming the delivery of education and training, pp. 183-194.

Sahin, A. R., et al. (2020). 2019 novel Coronavirus (COVID-19) out- break: A review of the current literature. EJMO, 4(1), 1-7.

Shariffuddin, S. A., Shaaidi, W. R. W., \& Hashim, S. M. (2017). Social networks as instructional tools beyond a classroom. International Journal of Advanced and Applied Sciences, 4(12), 185-192.

Sharma, P. (2010). Key concepts in ELT: Blended learning. ELT Journal, 64(4), 456-458.

Heather Staker and Michael B Horn (2012). Classifying K-12 blended learning. In: Innosight institute.

Carolyn N Stevenson (2018). Enhancing education through open degree pro- grams and prior learning assessment. IGI Global.

Melanie Swan (2015). Blockchain: Blueprint for a new economy. O’Reilly Media, Inc.

Kaye Thorne (2003). Blended learning: How to integrate online \& traditional learning. Kogan Page Publishers.

Tirziu, A.-M., \& Vrabie, C. (2015). Education 2.0: E-learning methods. Procedia-Social and Behavioral Sciences, 186, 376-380.

AV Tolbatov, SV Agadzhanova, and VA Tolbatov (2018). Using blockchain technology for E-learning, pp. 110-113. In: 1.

Ahmed Mohamed Fahmy Yousef, Ulrik Schroeder, and Marold Wosnitza (2015). Effective design of blended MOOC environments in higher education. Tech. rep. CiL Center for Innovative Learning Technologies.

Li Yuan and SJ Powell (2013). MOOCs and open education: Implications for higher education. In.

Yunus, M. M., Salehi, H., \& Chenzi, C. (2012). Integrating social networking tools into ESL writing classroom: Strengths and weak- nesses. English Language Teaching, 5(8), 42-48.

Publisher's note Springer Nature remains neutral with regard to jurisdictional claims in published maps and institutional affiliations.

\title{
Affiliations
}

\section{Anissa Cheriguene ${ }^{1,2} \cdot$ Taieb Kabache $^{2} \cdot$ Chaker Abdelaziz Kerrache $^{3} \cdot$ Carlos T. Calafate $^{4}$. Juan Carlos Cano ${ }^{4}$}

\author{
Anissa Cheriguene \\ cheranissa@gmail.com \\ Taieb Kabache \\ taibkabache@gmail.com \\ Carlos T. Calafate \\ calafate@disca.upv.es \\ Juan Carlos Cano \\ jucano@disca.upv.es
}


Department of English Language and Literature, University of Ouargla, Ouargla, Algeria

2 Department of English, ENS of Laghouat, Laghouat, Algeria

3 Laboratoire d'Informatique et de Mathématique, Université Amar Telidji de Laghouat, Po Box 7022, Oasis-Nord, Laghouat, Algeria

4 Department of Computer Engineering, Universitat Politècnica de València, Camino de Vera, S/N, Valencia, Spain 